\title{
Survival of Cancer Patients in Northeast China: Analysis of Sampled Cancers from Population-Based Cancer Registries
}

\section{Yanxia Li, MD ${ }^{1}$ \\ Liya $Y u, M D^{1}$ \\ Jun $\mathrm{Na}, \mathrm{MD}^{1}$ \\ Shuang Li, MD 1 \\ Li Liu, MD'1 \\ Huijuan Mu, MD'1 \\ Xuanjuan Bi, MD2 \\ Xiaoxia An, MD \\ Xun Li, MD ${ }^{4}$ \\ Wen Dong, MD \\ Guowei Pan, MD}

${ }^{1}$ Institute of Chronic Disease, Liaoning Provincial Center for Disease Control and Prevention, Shenyang, ${ }^{2}$ Institute of Chronic Disease, Anshan Municipal Center for Disease Control and Prevention, Anshan, ${ }^{3}$ Institute of Chronic Disease,

Benxi Municipal Center for Disease Control and Prevention, Benxi, Institute of Chronic Disease, Shenyang Municipal Center for Disease Control and Prevention, Shenyang, ${ }^{5}$ Department of Personal Resource,

Dalian Medical University, Dalian, China

\begin{abstract}
Purpose
The cancer survival was characterized by following up sampled subgroups of cancer cases from three population-based cancer registries in Northeast China.

\section{Materials and Methods}

Survival analysis was used to analyze 6,871 patients, who had one of the 21 most common cancers based on sampling from the population-based cancer registries of three cities in Liaoning Province. All patients were diagnosed between 2000 and 2002 and were followed up to the end of 2007 by active and passive methods. The 5-year age standardized relative survival rates (ASRS) were estimated for all cancers combined and each of the 21 individual cancers.
\end{abstract}

\section{Results}

The survival status was traced for $80.8 \%$ of 8,506 sampled cancer cases. The 5 -year ASRS for all 21 cancers combined was $41.5 \%$ (95\% confidence interval, 40.3 to 42.7 ), the highest ASRS was observed for thyroid cancer (85.2\%), breast cancer (78.9\%), uterine corpus cancer (75.9\%), and urinary bladder cancer (70.2\%); the lowest 5-year ASRS was noted in pancreatic cancer (8.8\%), liver cancer (11.0\%), esophageal cancer (18.8), and lung cancer (19.6\%). The cancer survival rates in Liaoning cities were similar to those of urban areas in mainland China, but significantly lower than those in Hong Kong, Korea, and Japan.

\section{Conclusion}

The strikingly poor cancer survival rates in three cities of Liaoning Province and in other places in China highlight the need for urgent investment in cancer prevention, early detection, and standardized and centralized treatment.

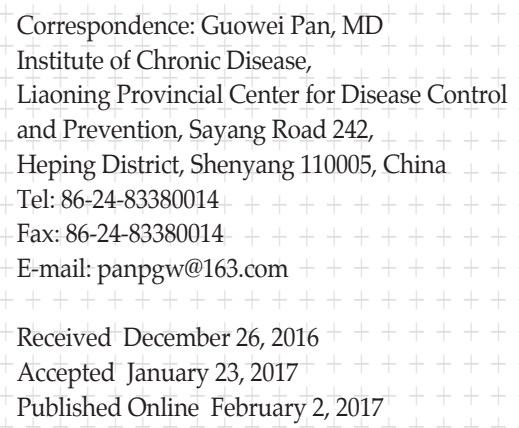

\section{Key words}

Neoplasms, Population-based study, Cancer registry, Survival rate, China

\section{Introduction}

Population-based cancer survival estimates reflect the general efficacy of cancer healthcare services and provide a key indicator of the progress in cancer control for a given region.
China has 3.09 million newly diagnosed cancer patients and 1.96 million cancer deaths each year, making effective cancer control an increasing challenge to the healthcare system in a country of 1.35 billion people [1,2]. Forty-four registries from 19 provinces provided data on the cancer incidence and mortality in China in 2011 [3], but population-based cancer sur- 
vival estimates are only available for three large cities (Hong Kong, Shanghai, and Tianjin) and one rural county (Qidong) [4]. A recent paper reported the first pooled-analysis of the cancer survival data of 2003-2005 in China [5], and found that Chinese cancer patients had a significantly lower age-standardized 5-year relative survival rate for all cancers combined $(30.9 \%)$ and most individual cancers relative to developed countries, with a marked disparity in the 5-year relative survival rate for individuals in urban regions (39.5\%) and rural regions (21.8\%). On the other hand, the low population representativeness (approximately $2 \%$ of the national population) and the limited geographic coverage provided by the 17 registries make it difficult to generalize these numbers to other areas of China, particularly for urban regions, because it only examined three large cities (Beijing, Dalian, and Zhongshan); more studies from other cities are warranted.

Similar to other developing countries, the incomplete or inaccurate personal identity information in the death and cancer registry certificates or records make it difficult to perform a passive follow-up of cancer patients in China, a procedure that is common in Western countries [6,7]. A study in India showed that passive follow-up alone results in a large upward bias for cancer survival estimates [8]. A high response rate is difficult to achieve when actively following up a large number of newly diagnosed cancer cases because this requires substantial finances, personnel, time, and other resources, particularly in China, which has approximately 3 million newly diagnosed cancers each year. Therefore, following up a sampled subgroup of a population-based cancer registry with passive and active follow-up methods may be a reasonable and cost-efficient method to estimate the cancer survival rates in the general population. Calculation of the age-standardized survival ratios (ASSR) makes it possible to estimate the survival rates of a target cancer population by following up the sampled cancer cases; these estimates are comparable to the survival data of other regions $[9,10]$. Previous studies have used this method to estimate and compare the cancer survival rates in the Philippines [11,12]. The aim of the present study was to provide the first estimate of the 5-year survival rates for 21 major cancers in the urban areas of Liaoning Province.

\section{Materials and Methods}

\section{Cancer registry in Liaoning}

The population-based cancer registry and death registry in the three cities of Liaoning Province was established by the Liaoning Provincial Center for Disease Control and Prevention (LNPCDCP) in 1982 and 1984, respectively. These registries used passive notification to register all cancer cases diagnosed in each city according to the Guidelines for Chinese Cancer Registration, which followed the rules of the International Agency for Research on Cancer. In the period, 2000 to 2005, the average annual incidence of cancer was 190 per 100,000 for males and 155 per 100,000 for females.

This study was approved by the ethics committee of Liaoning Provincial Center for Disease Control and Prevention.

\section{Selection of patients for follow-up}

To obtain representative and stable survival rate estimates for the major cancers in the urban population of Liaoning Province, randomly sampled subgroups of the 21 most common cancers from three of seven registries (Shenyang, Anshan, and Benxi) were actively followed up. These registries were selected based on the representativeness of socioeconomic development, quality of medical service, and population size.

The cancer registry database of three cities from 1 January 2000 to 31 December 2002 revealed 32,877 incident cases. The most common 21 cancers (accounting for $92.4 \%$ of all cases) were selected for the analysis ( 30,384 cases). This study used a sampling procedure similar to that used in following up subsamples of the cancer population in the Philippines [11,12]. At least 200 cases for of each sex were selected randomly for each individual cancer. For gender-specific cancers, 200 cases were selected for each sex in the database of 2000-2002. All cases were included if a specific cancer had fewer than 200 cases for each gender. A total of 8,506 cases were sampled, accounting for $28.0 \%$ of the 30,384 cases for the 21 cancers registered during 2000-2002 in the three cities.

\section{Follow up procedure}

A mixture of active and passive methods was used to follow up the survival status of the selected patients until December 31, 2007. First, the clinical follow-up notes were checked in each hospital where the patients had been diagnosed and treated to confirm the diagnosis and to collect vital information. Second, a passive follow-up was conducted by matching the selected cases with the records from the death registry database in the LNPCDPC. Third, all remaining cases were matched with the records in the personal identification card database from the police bureau of three cities, in which a unique PIN or a combination of personal identifiers were used by combining the first and last names, address, and date of birth. Fourth, an active follow-up by personal visits to the patients, their families, or work places at the last known place of residence/work was used to 
Table 1. Characteristics of the cancer patients sampled and analyzed in three cities of Liaoning Province, China from 20002002, with a follow-up to the end of 2007 by active and passive methods

\begin{tabular}{|c|c|c|c|c|c|c|c|c|}
\hline \multirow{2}{*}{ Cancer } & \multirow{2}{*}{ ICD-10 } & \multirow{2}{*}{$\begin{array}{c}\text { Incidence } \\
\text { No. }(\mathrm{n}=32,877)\end{array}$} & \multirow{2}{*}{ Sampled } & \multicolumn{3}{|c|}{ Un-analyzed } & \multirow{2}{*}{ Analyzed } & \multirow{2}{*}{$\begin{array}{l}\text { Histologically } \\
\text { verified }\end{array}$} \\
\hline & & & & $\mathrm{DCO}$ & Lost & Excluded & & \\
\hline Oral cavity & C00-14 & 493 & $360(73.0)$ & $25(6.9)$ & $54(15.0)$ & $6(1.7)$ & $275(76.4)$ & $228(82.9)$ \\
\hline Esophagus & C15 & 1,112 & $547(49.2)$ & $37(6.8)$ & 69 (12.6) & $10(1.8)$ & $431(78.8)$ & $287(66.6)$ \\
\hline Stomach & $\mathrm{C} 16$ & 3,283 & 475 (14.5) & $2(0.4)$ & $32(6.7)$ & $7(1.5)$ & 434 (91.4) & $353(81.3)$ \\
\hline Colon & $\mathrm{C} 18$ & 1,797 & $414(23.0)$ & 0 & $27(6.5)$ & $3(0.7)$ & 384 (92.8) & $323(84.1)$ \\
\hline Rectum & C19-21 & 2,278 & 442 (19.4) & 0 & $51(11.5)$ & $2(0.5)$ & $389(88.0)$ & 318 (81.7) \\
\hline Liver & $\mathrm{C} 22$ & 2,905 & 397 (13.7) & $3(0.8)$ & $31(7.8)$ & $17(4.3)$ & $346(87.2)$ & $115(33.2)$ \\
\hline Pancreas & $\mathrm{C} 25$ & 866 & $658(76.0)$ & $61(9.3)$ & $69(10.5)$ & $9(1.4)$ & $519(78.9)$ & $249(48.0)$ \\
\hline Lung & C33-34 & 8,647 & $561(6.5)$ & $3(0.5)$ & $44(7.8)$ & $12(2.1)$ & $502(89.5)$ & 315 (62.7) \\
\hline Bone & C40-41 & 330 & $236(71.5)$ & $21(8.9)$ & $33(14.0)$ & $57(24.2)$ & $125(53.0)$ & $81(64.8)$ \\
\hline Skin & C43-44 & 182 & $143(78.6)$ & $9(6.3)$ & $34(23.8)$ & $5(3.5)$ & $95(66.4)$ & $81(85.3)$ \\
\hline Breast & $\mathrm{C} 50$ & 3,075 & 360 (11.7) & $3(0.8)$ & $33(9.2)$ & $6(1.7)$ & 318 (88.3) & $296(93.1)$ \\
\hline Cervix & C53 & 512 & $360(70.3)$ & $9(2.5)$ & $58(16.1)$ & $22(6.1)$ & $271(75.3)$ & $243(89.7)$ \\
\hline Corpus uteri & C54-55 & 426 & 319 (74.9) & $14(4.4)$ & $52(16.3)$ & $8(2.5)$ & $245(76.8)$ & 235 (95.9) \\
\hline Ovary & $\mathrm{C} 56$ & 638 & $360(56.4)$ & $6(1.7)$ & $34(9.4)$ & $14(3.9)$ & $306(85.0)$ & $257(84.0)$ \\
\hline Prostate & C61 & 180 & $155(86.1)$ & $5(3.2)$ & $17(11.0)$ & $4(2.6)$ & $129(83.2)$ & $98(76.0)$ \\
\hline Kidney & C64 & 982 & $501(51.0)$ & $30(6.0)$ & $65(13.0)$ & $5(1.0)$ & $401(80.0)$ & $304(75.8)$ \\
\hline Urinary bladder & C67 & 604 & $526(87.1)$ & $23(4.4)$ & $63(12.0)$ & $7(1.3)$ & $433(82.3)$ & $370(85.5)$ \\
\hline Brain & C70-72 & 658 & $506(76.9)$ & $56(11.1)$ & $84(16.6)$ & $52(10.3)$ & $314(62.1)$ & $249(79.3)$ \\
\hline Thyroid & $\mathrm{C} 73$ & 302 & $253(83.8)$ & $6(2.4)$ & $36(14.2)$ & $7(2.8)$ & $204(80.6)$ & $179(87.7)$ \\
\hline $\begin{array}{l}\text { Lymphoma and } \\
\text { multiple myeloma }\end{array}$ & $\mathrm{C} 81$ & 494 & 407 (82.4) & $29(7.1)$ & $51(12.5)$ & 7 (1.7) & $320(78.6)$ & 155 (48.4) \\
\hline Leukemia & C91-95 & 620 & $526(84.8)$ & $47(8.9)$ & $48(9.1)$ & $1(0.2)$ & $430(81.7)$ & $367(85.3)$ \\
\hline 21 Cancers combined & & 30,384 & $8,506(28.0)$ & $389(4.6)$ & 985 (11.6) & $261(3.1)$ & $6,871(80.8)$ & $5,103(74.3)$ \\
\hline Other cancers & & $\begin{array}{l}2,493 \\
(7.58)\end{array}$ & - & - & - & - & - & - \\
\hline
\end{tabular}

Values are presented as number (\%). ICD-10, International Classification of Diseases, 10th edition; DCO, notification by death certificates only.

ascertain the survival of a patient whose status could not be confirmed by the record linkage.

The original sample of 21 selected cancers included 8,506 patients. The survival was traced and 6,871 cases $(80.8 \%)$ were finally included in the analysis. The list comprised 4,908 patients who died and 2,352 who were alive as of December 31,2007 . A total of 1,635 cases were excluded from the analysis due to loss to follow up ( $\mathrm{n}=985,11.6 \%)$, notification by death certificates only (DCO; $n=389,4.6 \%$ ), and problems in the final diagnosis $(n=261,3.1 \%)$.

\section{Statistical analysis}

\section{1) Age-standardization of the survival rates}

The relative survival rate was estimated as the ratio of the observed survival rate to the expected survival rate. In this study, the expected survival probabilities were estimated from age- and sex-specific abridged life tables of the three cities using the Ederer II method [13]. A direct standardization method [9] was used to estimate the relative survival rates of all eligible patients, the weights were equal to the inverse of the sampling proportion for each stratum of age and sex of the cancer in the corresponding database. The International Cancer Survival Standard weights [14] and the estimated incident cancer cases from developing countries in 2002 [15] were used as the standard cancer populations to calculate the ASSRs. This allowed a comparison of the present results with those of other countries or other areas in China [5,16-18]. All statistical analyses were performed using a specific program for the cancer survival in SAS software ver. $9.1[19]$. 
Table 2. Age standardized 5-year relative survival rates (\%) weighted to the original age and sex structure of the corresponding cancer in the 2000-2002 database for 21 cancers diagnosed from 2000-2002 in three cities of Liaoning Province, China

\begin{tabular}{|c|c|c|c|}
\hline \multirow{2}{*}{ Cancer } & \multicolumn{3}{|c|}{ Age-standardized survival ratio } \\
\hline & Male & Female & All \\
\hline Oral cavity & $39.2 \pm 3.4$ & $45.8 \pm 4.5$ & $41.6 \pm 2.7$ \\
\hline Esophagus & $17.0 \pm 2.0$ & $20.4 \pm 3.5$ & $17.8 \pm 1.7$ \\
\hline Stomach & $31.4 \pm 2.7$ & $35.2 \pm 3.4$ & $32.8 \pm 2.1$ \\
\hline Colon & $55.5 \pm 3.6$ & $57.2 \pm 3.4$ & $56.4 \pm 2.5$ \\
\hline Rectum & $56.3 \pm 3.3$ & $56.1 \pm 3.5$ & $56.2 \pm 2.4$ \\
\hline Liver & $8.8 \pm 1.6$ & $15.2 \pm 3.2$ & $10.7 \pm 1.5$ \\
\hline Pancreas & $8.7 \pm 1.5$ & $8.6 \pm 1.7$ & $8.6 \pm 1.1$ \\
\hline Lung & $18.1 \pm 2.2$ & $21.5 \pm 2.4$ & $19.7 \pm 1.6$ \\
\hline Bones & $33.5 \pm 5.2$ & $38.5 \pm 6.0$ & $35.8 \pm 4.0$ \\
\hline Skin & $46.5 \pm 6.5$ & $41.2 \pm 7.9$ & $44.3 \pm 5.0$ \\
\hline Breast & - & $78.9 \pm 2.2$ & $78.9 \pm 2.2$ \\
\hline Cervix & - & $59.0 \pm 2.6$ & $59.0 \pm 2.6$ \\
\hline Corpus uteri & - & $76.5 \pm 2.5$ & $76.5 \pm 2.5$ \\
\hline Ovary & - & $37.9 \pm 2.4$ & $37.9 \pm 2.4$ \\
\hline Prostate & $38.6 \pm 4.7$ & - & $38.6 \pm 4.7$ \\
\hline Urinary bladder & $71.9 \pm 3.0$ & $62.1 \pm 4.5$ & $69.3 \pm 2.5$ \\
\hline Kidney & $59.9 \pm 3.1$ & $59.8 \pm 3.9$ & $59.9 \pm 2.4$ \\
\hline Brain & $39.9 \pm 3.4$ & $45.5 \pm 3.7$ & $42.5 \pm 2.5$ \\
\hline Thyroid & $77.5 \pm 6.9$ & $85.1 \pm 2.7$ & $83.7 \pm 2.6$ \\
\hline Lymphoma and multiple myeloma & $32.8 \pm 3.0$ & $31.0 \pm 3.9$ & $32.1 \pm 2.4$ \\
\hline Leukemia & $21.5 \pm 2.4$ & $19.3 \pm 2.4$ & $20.5 \pm 1.7$ \\
\hline All 21 cancers & $34.8 \pm 0.8$ & $50.5 \pm 0.8$ & $43.4 \pm 0.6$ \\
\hline
\end{tabular}

Values are presented as mean \pm standard deviation.

Table 3. Age-standardized 5-year relative survival rates (\%, by estimated incident cancer cases from developing countries 2002 [15]) of cancer patients in three cities of Liaoning Province (2000-2002), and Hong Kong (1996-2001 [16]) and Incheon of Korea (1997-2001) [20]

\begin{tabular}{|c|c|c|c|c|c|}
\hline Cancer & Liaoning & Hong Kong & Difference & Incheon & Difference \\
\hline Prostate & 39.6 & 79.3 & -39.7 & 63.5 & -23.9 \\
\hline Melanoma of skin & 30.7 & 60.1 & -29.4 & 71.0 & -40.3 \\
\hline Ovary & 38.8 & 63.6 & -24.8 & 52.7 & -13.9 \\
\hline Cervix & 59.0 & 77.3 & -18.3 & 73.8 & -14.8 \\
\hline Liver & 11.0 & 22.4 & -11.4 & 16.0 & -5.0 \\
\hline Breast & 78.9 & 90.0 & -11.1 & 74.3 & 4.6 \\
\hline Thyroid & 85.2 & 93.7 & -8.5 & 90.0 & -4.8 \\
\hline Pancreas & 8.8 & 17.2 & -8.4 & 11.3 & -2.5 \\
\hline Corpus uteri & 75.9 & 84.1 & -8.2 & 82.2 & -6.3 \\
\hline Esophagus & 18.8 & 26.1 & -7.3 & 21.7 & -2.9 \\
\hline Urinary bladder & 70.2 & 75.9 & -5.7 & 65.9 & 4.3 \\
\hline Stomach & 33.4 & 38.7 & -5.3 & 42.9 & -9.5 \\
\hline Kidney & 60.3 & 65.5 & -5.2 & 64.4 & -4.1 \\
\hline Brain & 42.6 & 46.7 & -4.1 & 39.6 & 3.0 \\
\hline Lung & 19.6 & 22.1 & -2.5 & 18.4 & 1.2 \\
\hline
\end{tabular}

Difference: rate differences between Liaoning and Hang Kong [17] and Incheon, Korea [20]. 


\section{Results}

Table 1 lists the numbers and percentages of the 21 most common cancers that were followed up. A total of 8,506 sampled cases were included, which accounted for $28.0 \%$ of the 30,384 cases of the 21 major types of cancers registered during 2000-2002 in the three cities of Liaoning. The sampling rates ranged from $6.5 \%$ (lung cancer) to $86.1 \%$ (prostate cancer), and the overall percentage of cases registered as DCOs was $4.6 \%$. The survival status of 985 cases (11.6\%) could not be confirmed. Another 261 cases (3.1\%) were excluded because the final diagnoses were benign, uncertain, or borderline malignancy; in situ (except breast and bladder cancer); or metastatic to the index organ from elsewhere. Thus, $80.8 \%$ of 8,506 cases sampled were included to estimate the survival probability.

Table 2 lists the 5-year relative ASSRs adjusted to the age structure of each cancer in the original database. The most favorable 5-year ASSRs were thyroid cancer (83.7\%), breast cancer $(78.9 \%)$, uterine corpus cancer $(76.5 \%)$, urinary bladder cancer $(69.3 \%)$, and kidney cancer $(59.9 \%)$. The least favorable 5-year ASRRs were for pancreatic cancer $(8.6 \%)$, liver cancer $(10.7 \%)$, esophageal cancer $(17.8 \%)$, lung cancer (19.7\%), and leukemia (20.5\%). Females had better 5-year survival rates than males for most cancers, except for urinary bladder cancer, skin cancer, leukemia, lymphoma and multiple myeloma, pancreatic cancer, and rectal cancer.

Table 3 compares the 5-year relative ASSRs of the cancer patients from Liaoning cities (2000-2001), Hong Kong (19962001) [16], and Incheon, Korea (1997-2001) [20]; all were adjusted to the estimated age structure of the incident cancer cases from developing countries in 2002 [15]. The patients from Liaoning had significantly lower survival rates than those from Hong Kong for all 15 comparable cancers, and these differences ranged from $-2.5 \%$ (lung cancer) to $-39.7 \%$ (prostate cancer). They also had significantly lower survival rates than those from Incheon, Korea for 11 out of 15 cancers, except for breast cancer, urinary bladder cancer, brain cancer, and lung cancer.

Table 4 lists the 5-year relative ASSRs adjusted for age with the International Cancer Survival Standard weights [14] of six selected cancers and all cancers among three cities in Liaoning (2000-2002) and the urban and rural areas of China (2003-2005) [5] and Japan (1997-1999) [21]. The 5-year relative ASSRs were comparable in the three cities and urban areas (three cities) of China, but were significantly lower than those of Japan.

\section{Discussion}

To the best of the authors' knowledge, this is the first report of the population-based cancer survival estimates of major cancers in Northeast China. Considerable effort was made to follow up $80.8 \%$ of the 8,506 sampled cancer patients. A total of $11.6 \%$ of patients were lost to follow up. This which is similar to two studies in the Philippines, which had follow up rates of $78.0 \%$ and $77.9 \%$, respectively $[11,12]$. A total of 6,871 patients were included in the final analysis, and $4,519(65.8 \%)$ of these patients died by the end of the followup. A passive follow-up by linkage to the death database only confirmed 2,434 of the 4,519 deaths (53.9\%). Additional follow-up (checking the medical records in hospitals: 340 deaths; personal identification database, 1,239 deaths; and home visits, 517 deaths) confirmed another 2,096 deaths $(42.7 \%)$. This supports the findings of a study in India [8] in that the use of passive case follow-up alone will lead to a serious upward bias for the cancer survival estimates.

The developing country 2002 weighted 5-year cancer survival rates for Liaoning cities were significantly lower than that of Hong Kong [16] for all 15 cancers (Table 3) and

Table 4. Age-standardized 5-year relative survival rates (\%, by international standard cancer patient population [16]) of cancer patients in three cities of Liaoning Province, and urban China [5] and Japan (1997-1999) [21]

\begin{tabular}{|c|c|c|c|c|c|c|}
\hline \multirow{2}{*}{ Cancer } & \multicolumn{3}{|c|}{ Male } & \multicolumn{3}{|c|}{ Female } \\
\hline & Liaoning & Urban China & Japan & Liaoning & Urban China & Japan \\
\hline Esophagus & 17.4 & 18.9 & 30.7 & 21.2 & 23.6 & 37.3 \\
\hline Stomach & 31.5 & 31.8 & 62.6 & 34.4 & 34.3 & 61.2 \\
\hline Colorectum & 56.0 & 51.8 & 68.4 & 56.7 & 50.5 & 65.5 \\
\hline Liver & 8.8 & 16.1 & 23.7 & 15.3 & 16.8 & 21.8 \\
\hline Lung & 17.9 & 19.0 & 22.4 & 21.6 & 20.5 & 33.5 \\
\hline Breast & - & - & - & 77.7 & 77.8 & 85.5 \\
\hline All & 35.1 & 33.9 & 50.0 & 49.9 & 45.3 & 59.8 \\
\hline
\end{tabular}


Incheon, Korea for 11 out of 15 cancers [20]. The largest differences in survival were for prostate cancer (39.6\% vs. $79.3 \%)$, melanoma ( $30.7 \%$ vs. $60.1 \%)$, and ovarian cancer (38.8\% vs. 63.6\%). The smallest differences were for lung cancer ( $19.6 \%$ vs. $22.1 \%)$, brain cancer ( $42.6 \%$ vs. $46.7 \%$ ), and kidney cancer ( 60.3 vs. $65.5 \%)$. Because of the different follow-up times used in the present study (2000-2002 cases followed up until 2007) and the Hong Kong study (1996-2001 cases followed up until 2003) [16], the real difference in cancer survival rates between these two regions should be considered an underestimate. These results are also consistent with the findings that China had significantly lower cancer survival rates than developed countries [5]. China has substantial inter- and intra-regional inequality in socioeconomic development, healthcare coverage, and healthcare affordability [22]. Wide differences in cancer survival rates can be found between countries or regions with different gross national incomes (GNI) per person [23]. Therefore, the finding that the cancer survival rates are best in Hong Kong, intermediate in urban China and Liaoning cities, and poor in rural China $[5,16]$, or best in Hong Kong, intermediate in Shanghai, and poor in Qidong [16-18] (depending on the type of cancer) is expected because this reflects the per person GNIs of different regions of China. This reflects the large differences in the efficacy of cancer control and treatment in a country with serious healthcare inequalities [22].

Compared to the results of a recent population-based study in China [5], three Liaoning cities and urban China overall (three cities) had similar 5-year survival rates for all cancers combined (43.2\% vs. $39.5 \%$ ) as well as for individual cancers, but all were significantly lower than the corresponding rates of Japan [21]. This highlights the need to obtain reliable estimates of the cancer survival rates by following up a sampled subgroup of a population-based cancer registry with passive and active follow-up methods in developing countries, such as China, India, and the Philippines $[4,8,11,12]$.

As expected, significantly decreasing cancer survival rates with increasing age and stage at diagnosis for all cancers were observed (data not shown). A few organized cancer screening programs in Liaoning Province operated during the period covered by this study. A 2009 survey by the present author (unpublished) showed that only $9.2 \%$ of citizens over 18 years of age in Liaoning province have ever participated in cancer screening examinations in the past 5 years. In addition, $14.2 \%$ of breast cancer patients, $17.6 \%$ of cervical cancer patients, and $25.8 \%$ of ovarian cancer patients were at stage I at diagnosis, which is significantly lower than the rates for Hong Kong $(22.2 \%, 31.9 \%$, and $42.0 \%$, respectively) [24-26]. The lower cancer screening rates and delayed diagnosis for patients in the present study provides striking evidence that the lack access to quality healthcare contributes to the significant inequality in survival rates for different regions of China [2,5].

Previous studies showed that the standard treatment protocols provide the best treatment based on previous clinical trials and that the centralization of hospitals for the diagnosis and treatment of cancer is associated with the better delivery of appropriate therapies [2]. A survey of six cities of Liaoning Province in 2004 showed that only $32.5 \%$ of all cancer patients received standard therapy, ranging from $3.6 \%$ for gallbladder cancer to $52.6 \%$ for breast cancer between 1991 and 2000 [27]. This was significantly lower than the corresponding rate of $82.2 \%$ in Shanghai [27]. Large variations occurred in the introduction and implementation of standard therapy protocols for different types of hospitals: $46.8 \%$ for cancer specific hospitals attached to municipal hospitals, $33.5 \%$ for cancer departments of hospitals at the municipal or county levels, and $3.3 \%$ for other hospitals without cancer departments $[27,28]$. The lower levels of standardized and centralized treatment are related to the limitations in personnel, training, facilities, management, and economic factors, which likely contributed to the lower cancer survival rate in Liaoning Province.

Some limitations should be considered in interpreting these cancer survival estimates. First, the 5-year survival rates for 21 major cancers were estimated by following up a sampled subgroup of a population-based cancer registry, rather than all cases in the registry. On the other hand, the random samples from the population-based registries should reasonably represent the distribution of all cases in the registry. Compared to the large bias introduced when using passive follow-up alone in places with limited resources, such as China and other developing countries, it is believed that following up a sampled subgroup using passive and active methods together provides a higher response rate and a more reliable estimate $[8,11,12]$. Although sampling errors would bias the estimations to some extent, the use of age standardization and assessments of the relative survival reduce the impact of age differences and make the survival estimates more comparable with other data. Therefore, the present results can serve as a baseline for subsequent comparisons that seek to assess any improvements in cancer survival. Second, although considerable efforts were made to actively follow-up the sampled patients, 391 cases (11.6\%) were lost to follow-up for a variety of reasons, primarily high population migration and mobility. The cases lost to followup were assumed to have not received better treatment than those who were traced, so the reported survival rates should be considered as overestimates. Third, the information on the distribution of cancer stages was incomplete and not fully comparable for most cancers in the four cancer registries. This limited the ability to explore the issues underlying the survival differences in more detail. Nevertheless, the pooled 
data for each group showed decreasing survival rates with increasing stages for all cancers, adding strength to the data quality in this study.

The remarkably lower cancer survival rates in the urban areas of Liaoning Province relative to Hong Kong, Korea, and Japan highlight the need for urgent and adequate investment in comprehensive cancer control for Liaoning Province. In addition, to improve the accessibility and affordability of healthcare services for the general population, efforts are needed to improve public and professional awareness, early detection, and prompt treatment using standardized and centralized care. Provincial cancer-control policies should focus on balanced investments in the prevention, early detection, and treatment of the most common cancers, and should also improve the completeness of the populationbased cancer survival statistics using passive and active methods. Considering the limited resources and the difficulty in rapidly improving the quality of the cancer registry and population database, a high-quality active follow-up of sampled cases is a reasonable, cost-effective, and reliable method for estimating cancer survival in China and other developing countries.

\section{Conflicts of Interest}

Conflict of interest relevant to this article was not reported.

\section{Acknowledgments}

The authors wish to thank the doctors and officers from the municipal centers for disease control and prevention, and the police bureau who were responsible for data collection, entry and processing. They also thank the source hospitals and vital statistics division that provided data to the registry.

This study was supported by the Liaoning Provincial Department of Public Health. The funders had no role in study design, data collection and analysis, decision to publish, or preparation of the manuscript.

\section{References}

1. Chen W, Zheng R, Zhang S, Zhao P, Zeng H, Zou X. Report of cancer incidence and mortality in China, 2010. Ann Transl Med. 2014;2:61.

2. Goss PE, Strasser-Weippl K, Lee-Bychkovsky BL, Fan L, Li J, Chavarri-Guerra $Y$, et al. Challenges to effective cancer control in China, India, and Russia. Lancet Oncol. 2014;15:489-538.

3. Zhao P, Chen W, Kong L. Cancer incidence and mortality in China 2003-2007. Beijing: Military Medical Science Press; 2011.

4. Sankaranarayanan R. Cancer survival in Africa, Asia, the Caribbean and Central America. Introduction. IARC Sci Publ. 2011;(162):1-5

5. Zeng H, Zheng R, Guo Y, Zhang S, Zou X, Wang N, et al. Cancer survival in China, 2003-2005: a population-based study. Int J Cancer. 2015;136:1921-30.

6. Berrino F, Sant M, Verdecchia A, Capocaccia R, Hakulinen T, Esteve J, et al. Survival of cancer patients in Europe. The Eurocare Study. IARC Scientific Publications No. 132. Lyon: IARC Press; 1995.

7. Sankaranarayanan R, Black RJ, Parkin DM. Cancer survival in developing countries. IARC Scientific Publications No. 145. Lyon: IARC Press; 1998.

8. Swaminathan R, Rama R, Shanta V. Lack of active follow-up of cancer patients in Chennai, India: implications for population-based survival estimates. Bull World Health Organ. 2008;86:509-15.

9. Parkin DM, Hakulinen T. Cancer registration, principles and methods: analysis of survival. IARC Sci Publ. 1991;(95):159-76.

10. Brenner H, Arndt V, Gefeller O, Hakulinen T. An alternative approach to age adjustment of cancer survival rates. Eur J Can- cer. 2004;40:2317-22.

11. Redaniel MT, Laudico A, Mirasol-Lumague MR, Gondos A, Pulte D, Mapua C, et al. Cancer survival discrepancies in developed and developing countries: comparisons between the Philippines and the United States. Br J Cancer. 2009;100: 858-62.

12. Redaniel MT, Laudico A, Mirasol-Lumague MR, Gondos A, Brenner H. Cancer survival differences between European countries and an urban population from the Philippines. Eur J Public Health. 2011;21:221-8.

13. Ederer F, Heise H. Instructions to IBM 650 programmers in processing survival computations. Methodological note No. 10. Bethesda, MD: National Cancer Institute; 1959.

14. Corazziari I, Quinn M, Capocaccia R. Standard cancer patient population for age standardising survival ratios. Eur J Cancer. 2004;40:2307-16.

15. Ferlay J, Bray F, Pisani P, Parkin DM. GLOBOCAN 2002: cancer incidence, mortality and prevalence worldwide, version 2.0. IARC CancerBase No. 5. Lyon: IARC Press; 2004.

16. Law SC, Mang OW. Cancer survival in Hong Kong SAR, China, 1996-2001. IARC Sci Publ. 2011;(162):33-41.

17. Xiang YB, Jin F, Gao YT. Cancer survival in Shanghai, China, 1992-1995. IARC Sci Publ. 2011;(162):55-68.

18. Chen JG, Zhu J, Zhang YH, Lu JH. Cancer survival in Qi Dong, China, 1992-2000. IARC Sci Publ. 2011;(162):45-53.

19. Brenner H, Gefeller O, Hakulinen T. A computer program for period analysis of cancer patient survival. Eur J Cancer. 2002;38:690-5.

20. Woo ZH, Hong YC, Kim WC, Pu YK. Cancer survival in 
Incheon, Republic of Korea, 1997-2001. IARC Sci Publ. 2011;(162):163-9.

21. Matsuda T, Ajiki W, Marugame T, Ioka A, Tsukuma H, Sobue $\mathrm{T}$, et al. Population-based survival of cancer patients diagnosed between 1993 and 1999 in Japan: a chronological and international comparative study. Jpn J Clin Oncol. 2011;41: 40-51.

22. Liu Y, Rao K, Wu J, Gakidou E. China's health system performance. Lancet. 2008;372:1914-23.

23. Sankaranarayanan R, Swaminathan R, Brenner $H$, Chen $K$, Chia KS, Chen JG, et al. Cancer survival in Africa, Asia, and Central America: a population-based study. Lancet Oncol. 2010;11:165-73.

24. Kwong A, Mang OW, Wong CH, Chau WW, Law SC; Hong Kong Breast Cancer Research Group. Breast cancer in Hong Kong, Southern China: the first population-based analysis of epidemiological characteristics, stage-specific, cancer-specific, and disease-free survival in breast cancer patients: 1997-2001. Ann Surg Oncol. 2011;18:3072-8.

25. Cheung FY, Mang OW, Law SC. A population-based analysis of incidence, mortality, and stage-specific survival of cervical cancer patients in Hong Kong: 1997-2006. Hong Kong Med J. 2011;17:89-95.

26. Wong KH, Mang OW, Au KH, Law SC. Incidence, mortality, and survival trends of ovarian cancer in Hong Kong, 1997 to 2006: a population-based study. Hong Kong Med J. 2012;18: 466-74.

27. Zhao Y, Dong W, Sun L, Xiao W, Pan T. A sampling investigation on executing the diagnosis and management norm for cancer in Liaoning province. Bull Chin Cancer. 2004;13:12-4.

28. Li DL, Zheng Y, Chen HQ, Xu YC, Lu W. Evaluation of implement of the diagnosis and treatment criterion for common cancer in China in Shanghai. Bull Chin Cancer. 2001;10:192-5. 